\title{
The Appleton Consensus: suggested international guidelines for decisions to forego medical treatment
}

\author{
John M Stanley Lawrence University, and 34 others
}

\section{Author's abstract}

Thirty-three physicians ${ }^{\star}$, bioethicists, and medical economists from ten different countries met at Lawrence University, Appleton, Wisconsin, to create The Appleton Consensus: International Guidelines for Decisions to Forego Medical Treatment. The guidelines deal with four specific decision-making circumstances:

\section{Five guidelines were created for decisions involving} competent patients or patients who have executed an advance directive before becoming incompetent, and those guidelines fell into three categories.

2. Thirteen guidelines were created for decisions involving patients who were once competent, but are not now competent, who have not executed an advance directive.

3. Seven guidelines were created for decisions involving patients who are not now and never have been competent, for whom 'no substituted judgement' can be rendered.

4. Eleven guidelines were created for decisions involving the scarcity of medical resources, which exists in all communities. Five concepts were identified as being critical in the establishment of priorities, given the reality of scarce health resources (1).

* The term 'physician' is used in the American sense, synonymous with 'medical practitioner'.

Editor.

\section{Introduction}

Thirty-three delegates from ten different countries (2) convened in Appleton, Wisconsin, USA, from May 15-19, 1988, under the auspices of the Lawrence University Program in Biomedical Ethics, to create a first draft of what they hoped might become international guidelines for treatment abatement procedures. The delegates attending committed themselves to convene a forum at an appropriate level in their home country or community for further discussion of the statement produced at the conference.

\section{Key words}

Foregoing treatment; autonomy; euthanasia; advance directives; scarcity; persistent vegetative state.
Twenty-five of the thirty-three delegates were physicians, representing eleven different medical $\vec{G}$ specialisations. Of the eight non-physicians, six had $\omega$ extensive clinical experience in ethics consultations. $\vec{N}$ One of the eight was a medical economist. Seven were ${ }^{0}$ bioethicists: two with backgrounds in philosophy, two in theology, two in law, and one in comparative religion and ethics. Several of the delegates had had $\Phi$ experience in the creation of important medical ethics $\frac{\overline{\mathbb{D}}}{\mathrm{D}}$ guidelines in the past.

Twenty-six of the thirty-three delegates had met吕 together in a preparatory conference one year earlier. Seven of the delegates were new (3).

Two particular sets of concerns had emerged in $e^{\circ}$ first Appleton working conference: 1) concex regarding decisions to forego medical treatme including life-prolonging treatment, precipitated by autonomous requests by patients or their surrogates, and 2) concerns regarding decisions to forego medical $\stackrel{\mathbb{Q}}{\complement}$ treatment as a result of pressures due to scarcity.

The goal of the second international conference was 3 to draft a statement addressing these concerns in the form of guidelines for discussion in medical ando medical ethics communities internationally.

The delegates were divided into four working groups (4). In the first three plenary sessions of the conference, the delegates studied existing sets of $\frac{}{-}$. guidelines (5), discussed the processes of searching forô consensus, and set their operational goals for the working sessions. After four working sessions, each음 working group reported its progress for plenary discussion. During two further group sessions, the working groups reworked their statements in light of the suggestions from the plenary session before preparing a final report to the whole group. Each $\tilde{S}^{\circ}$ report was then discussed line by line in two long plenary sessions to produce the following statement.

\section{Preamble: ethical background}

In caring for patients, physicians, as individuals and as a profession, should act with integrity in providing medical treatment within certain norms of care and? concern. Despite widely diverse national, cultural $\underset{\Omega}{\Phi}$ religious, and political traditions, four prima facio moral values or principles summarise these norms (6). 
1. Autonomy All persons have a prima facie moral obligation to respect each other's autonomy insofar as such respect is compatible with the respect for the autonomy of all affected. This principle requires respect for patients' deliberated choices made in accordance with their own values, consciences, and religious convictions. Respect for patients' dignity and integrity, for honesty, and for promise-keeping are aspects of respect for autonomy. Respect for the autonomy of health care professionals is no less important and no more important than the respect for the autonomy of their patients.

2. Non-maleficence All persons have a prima facie moral obligation not to harm each other. The infliction or risking of harm to others, including the risks of medical practice, can only be justified by the pursuit of other moral values - principally benefits to patients sufficient to outweigh the harm.

3. Beneficence All persons have some moral obligation to benefit others, to some degree, including, perhaps even especially, those in need. The extent and scope of this obligation are, however, highly influenced by both cultural and individual interpretation. In any case, health care providers acknowledge themselves to have a particular obligation to benefit their patients and to do so with minimal harm.

4. Justice All persons have a prima facie moral obligation to act justly or fairly to others in the context of the distribution of scarce resources, in the context of respecting each other's rights, and in the context of obeying morally acceptable laws. Interpretation of the precise nature and extent of these obligations is highly dependent on both cultural and individual perspectives.

These four principles or values do not comprise a single ethical theory. Indeed, they often conflict and require interpretation and balancing. The four principles are given different weight in different cultures and some cultures would wish to add additional principles or values (7). Moreover, substantive disagreements exist within cultures about both their scope and relative weights.

Nonetheless, their acknowledgment provides a valuable cross-cultural basis for medico-moral analysis, discussion, and decision-making.

\section{Part I: For decisions involving competent (8) patients or patients who have executed an advance directive before becoming incompetent}

In the context of the norms of medical practice summarised in the preamble above, five guidelines are suggested concerning requests from competent patients or from incompetent patients who had competently provided advance directives, oral or written. These guidelines fall into three categories.

Guidelines

REFUSAL OF TREATMENT
1. If a competent patient rejects treatment that the physician believes to be in the patient's interests, especially where such treatments are life-prolonging, the physician should seek to explore the patient's reasons for such refusal and seek to correct any misunderstandings. However, a physician should not impose treatment if rejected (even if the treatment is potentially life-prolonging) and should explore alternatives that might be acceptable to the patient, including transfer of the patient to the care of a physician or institution prepared to respect the patient's wishes (9). In all cases including those where a patient's refusal of a specific treatment is respected, the physician and the health care institution have the obligation to continue to offer supportive care and treatment for pain and suffering.

2. Where an incompetent patient previously has given a competent advance directive to refuse treatment and/ or appointed a representative to make decisions about refusal of treatment, such advance directives and decisions should be respected by physicians and other health care workers.

REQUESTS FOR TREATMENT, INCLUDING LIFEPROLONGING TREATMENT

3. Physicians also have a strong prima facie obligation to respect competent patients' requests for lifeprolonging treatment. However, certain qualifications are relevant:

(a) Physicians are not obliged to provide physiologically futile treatments (ie treatments that cannot produce the desired physiological change). Where a physician considers a life-prolonging treatment not to be physiologically futile, but nonetheless 'futile' in the normal sense of the word because of the low probability of success or because of the low quality of life that would remain, then decisions about the withholding or withdrawal of such treatments should be made in the context of full and open discussion of the nature and extent of the 'futility' of the treatment with the patient or the patient's representative;

(b) If a requested treatment entails, according to the norms of medical practice, loss of function, mutilation, or pain disproportionate to benefit, the physician is not obliged to provide it;

(c) If a physician has a conscientious objection to a requested treatment, that physician is not obliged to provide it. The physician should explain all treatment options and his or her position regarding them. If the patient wishes, the physician should arrange an orderly transition to another physician of the patient's choice; (d) Scarcity of resources may sometimes require overriding a patient's request for a life-prolonging treatment (see Part IV).

4. Where an incompetent patient has previously given a competent advance directive requesting lifeprolonging treatment(s) and/or appointed a 
representative to make such requests, physicians have a strong prima facie obligation to respect such requests. The same qualifications apply here as in \#3 above.

\section{REQUESTS FOR INTERVENTIONS INTENDED} TO TERMINATE LIFE (VOLUNTARY EUTHANASIA)

5. Requests for euthanasia by competent patients severely and irremediably suffering as a result of incurable disease may be justified. It is a separate question whether they should be honoured. Physicians have an obligation to try to provide a peaceful, dignified, and humane death with minimal suffering. However, statutory legalisation of the intentional killing of patients by physicians is against the public interest (10). ${ }^{\star}$ Delegates disagreed as to whether physicians should, as in Holland, be protected from prosecution if euthanasia were carried out according to agreed guidelines.

\section{Part II: For decisions involving patients who were once competent, but are not now competent, who have not executed an advance directive}

These guidelines pertain to situations involving patients who once were but are no longer competent, who left no advance directive, and who have at least two potential future courses of life depending upon a treatment choice. That choice may be either: 1) whether to forego rather than use a particular treatment, or 2) which of several possible alternative treatments should be used.

\section{Guidelines}

\section{FULL MEDICAL PROGNOSIS}

The physician has the responsibility to discern, to the extent possible, the patient's current medical and social situation, the likely future course of the disease or condition in the absence of intervention, the full range of potentially useful interventions, and the likely course with each of these.

\section{PATIENT'S VALUES HISTORY}

The physician also has the obligation to ensure insofar as possible that the patient's own values and preferences in regard to the current situation are ascertained.

\section{DUTY TO INFORM}

Information about all alternatives that might be beneficial to the patient should be shared with the patient's family. The term 'family' should be understood to include those persons who are available and competent, have been involved with and concerned about the patient, are knowledgeable about the patient's values and preferences, and are willing to apply the patient's values to making the decision. This term might well include persons not related to the patient and might exclude relatives.

* Indicates dissent. Dissents are located at the end of the statement.

\section{4. 'SUBSTITUTED JUDGEMENT' DECISIONS}

If the physician can determine that a particular plan of care, including the foregoing of particular treatment, ises? clearly most in accord with the patient's values and if the patient's family and direct caregivers concur, then? that plan of care should be pursued.

\section{5. 'BEST INTERESTS' DECISIONS}

If the comparative merits of the alternative futures, in the light of the patient's values, do not clearly indicates which plan of care the patient would have preferred, then the physician, in consultation with the family, if available, and other direct care givers, should identify $\vec{\omega}$ the plan of care that would most generally be thoughto to advance most such patients' interests; and, if family and direct caregivers concur, it should be implemented. Ordinarily, for example, persons woulder want to preserve identity, be able to maintain independence and control, be able to interact with ${ }_{0}^{N}$ others, have pleasurable experiences, avoid pain ando suffering, and avoid being a severe burden upon others. Normally treatment must be justified in thesecs terms (11).

\section{DISCORD}

If there is a conflict between the responsible physician and an involved care giver or family member as to which course of care should be pursued, thên nọ procedures must be in place to ensure adequate attention to resolving this discord. Counselliwis discussion, consultation, and other informa interventions may bring about significant degrees of agreement (12). If the person(s) who disagree(s) witho the physician's recommendation is emotionally and socially distant and there are others who are $\overrightarrow{5}$ emotionally and socially close, then the physician may 3 disregard the claims of the more tangential party However, if the disagreement is with someone close to? the patient, the physician should not generally overrides that view without resorting to more formal conflict resolution processes. These might include intra윽 institutional authorities (for example, ethics. committees or department heads or administrators) of extra-institutional authorities (for example, the courts). Institutions and programmes of care should have available reliable and responsive procedures that ensure that all relevant considerations are given theire. due.

\section{SOCIALLY ISOLATED PATIENTS}

If the now-incompetent patient has no family of friends, the physician has an especially weighty obligation to ensure that decisions are made well. Nob all such patients need personal advocates (for example guardianships, ombudspersons, public officials), bue ${ }^{+}$ the physician should consult widely with other directo care givers, consultants, and relevant religious advisors. Some cases may merit formal review either by intra-institutional or extra-institutional authorities before the decision is made by the physician. The need for this prospective review should reflect the degree to 
which the decision is one with serious and irreversible effects, one with unavoidable uncertainties, one concerning a patient of a group with a history of being treated in a discriminatry manner, or one which is without substantial precedent.

\section{RESTORATION OF COMPETENCE}

When it is reasonable to believe that a patient could regain competence before a weighty decision must be made, the decision should be delayed in order to allow the patient the opportunity to make the decision.

\section{FUTILE TREATMENT}

A treatment that cannot reasonably be expected to achieve even its physiological objective is physiologically futile and need not be offered nor provided if requested (13).

\section{CARE PLAN CONSIDERATIONS}

Plans of care must be reasonably comprehensive, including considerations of what treatments to utilise, how long to employ them, and when and how to stop. Planned trials of one or more courses of care for individual patients are often very useful in delineating the likely course of the patient's response to treatment and should be encouraged. Withdrawing treatment already initiated should not be regarded as any more problematic, ethically speaking, than withholding such treatment initially. Indeed, often, some medical evidence is clearer after a trial of treatment, and withdrawing ineffectual or harmful treatment then has even more justification than would have withholding the treatment originally.

\section{QUALITY REVIEW}

The decision-making process must be documented and justified in writing to facilitate regular audit by the profession and others who may be involved in quality assurance processes.

\section{ACTIVE EUTHANASIA}

Intervention with the sole intention of causing death (as distinguished from foregoing treatment that is deemed inappropriate) has no place in the treatment of permanently incapacitated patients. However, vigorous treatment to relieve pain and suffering may well be justified, even if these interventions lead to an earlier death.

\section{PERSISTENT VEGETATIVE STATE}

The patient who is reliably diagnosed as being in the persistent vegetative state (PVS) has no self-regarding interests. Unless the patient in the past has requested or the family or caregivers now can justify continuing life-sustaining medical treatments, there is no reason to use those treatments $(14) .^{\star}$

Part III: For decisions involving patients who are not now and never have been competent

These guidelines address patients who now lack and have always lacked the capacity to choose for themselves with regard to life-prolonging medical treatment. They are patients for whom no 'substituted judgement' can be rendered, as their present or previous wishes and desires cannot be known. Within this group two further distinctions may be useful:

1. Between those who, due to anomaly, illness, or injury, will never develop decisional capacity in the future (such as anencephalics, the permanently unconscious, and the severely and permanently incapacitated) and those who can be anticipated, if they survive, to develop that capacity to varying degrees;

2. Between those who have a natural or agreed surrogate (for example, parents or guardian) and those who lack such a surrogate.

\section{Guidelines}

1. Regard for the value of life does not imply an absolute duty to employ life-prolonging treatment for non-competent patients. In setting reasonable limits for such treatment, third person judgements about quality of life (15), are inevitable. Responsible third person quality-of-life judgements consider, insofar as possible, how the options must appear from the perspective of one in the patient's condition and determine what would most generally be thought to count as quality for most such patients (16).

2. Assessing quality of life of these patients for purposes of medical decisions involves weighing the ratio of benefits and burdens (17).

3. In most decisions involving patients in this category, at least five sets of interests may be discerned:

a) the patient's;

b) the surrogate's or family's;

c) the physician's and those of other care givers;

d) the health care institution's (where continuing or withholding treatment may have religious, financial, and legal implications and may expose it to local or national publicity);

e) society's (including both the use of economic resources and the need for research to help future patients).

Normally, the patient's interests should be regarded as paramount. However, difficult moral dilemmas arise when the patient's interests are unclear or clearly conflict with a number of other interests. Societies differ in their preferences for mechanisms for arbitrating conflict in these difficult cases (for example, institutional ethics committee, courts). It is important to remember, however, that in the cases most commonly encountered, the various interests are not necessarily in conflict.

Often the patient's own interest is integrally interwoven with the interest of the family and the community. Part of the physician's clinical wisdom consists of responsibly weighing interests and creatively resolving apparently irreconcilable conflicts. 4. When the patient has a surrogate, the physician's obligation to the patient also requires certain duties towards the surrogate. These include: a) providing accurate information about the specific clinical 
problems; b) being honest; c) applying skills in effective communication; d) being willing to answer any questions that are asked; e) being aware of broader social and moral implications.

To act in a way that recognises these duties to the surrogate is to be worthy of the trust that one hopes the surrogate will place in the physician, so that a policy of mutual and shared decision-making may be fostered. When the patient is a child with an emerging capacity to participate in the decision-making process, both physician and surrogate demonstrate respect by responding to questions and concerns at a level consistent with the child's cognitive and moral development.

5. While the physician is required to act in a trustworthy manner towards the patient and surrogate, the range of interests that could conflict (see Part II, \#3 above) demands that that same standard of trustworthiness be translated to the level of social review and professional peer relationships. Whatever patterns an individual medical culture may employ to achieve that translation of standards, the process will be enhanced by careful documentation of medical care to facilitate thoughtful audit. This careful documentation should include both the careful recording of management plans and the internal reasoning that led to them. It should routinely include both medical evidence and the applications of principles which logically lead to the conclusions made about management. Such patterns of careful thinking and careful documentation constitute good clinical practice. How often audit occurs and by whom may be a matter of considerable difference among various countries. Adding extra layers of mandatory audit may compromise the quality of patient care without helping to avoid the occasional bad decision.

6. When patients lack a surrogate, little difficulty arises when the benefit-burden ratio clearly favours administration and continuation of life-prolonging treatment. When the benefit-burden ratio is less certain or reversed, a wide variety of mechanisms have been proposed to aid or to review the physician's decision-making (18).

7. The physician may appropriately withdraw or withhold life-prolonging treatment when, in the view of the informed surrogate and physician, continued treatment would lead to unacceptable burdens without sufficient compensating benefits. What counts as a benefit and a burden and the relative ratio between them depend on specific situational factors, and therefore, good decisions in this category of patients demand individual discretion. While these patients possess a vulnerability which makes them frequently subject to social discrimination and stigmatisation, their interests are not protected by the elimination of decisional discretion. On the contrary, a trustworthy physician and the processes of appropriate audit are better means of protecting the interests of vulnerable patients.

\section{Part IV: Scarcity (19)}

Growing needs and demands, a growing range of increasingly costly medical options, and diminishing? resources compel us to recognise that it is not feasible to offer all beneficial treatments that are medicallyo possible to all patients. Necessarily, all communities face scarcities. Some instances of scarcity can be addressed by a particular allocation of funds. Others, $\mathbb{\Phi}$ such as a shortage of organs for transplant, may involve absolute limitations whch may not be resolved easilye within the apparently acceptable range of ethical $\vec{O}$ choices. Scarcity, by definition, requires choice, and $\overrightarrow{-}$ any choice in the context of scarcity requires foregoing $\omega^{\omega}$ alternative choices. Societies may deny that they make such choices or disguise the ones they make, but they do so at the price of honesty, justice, and efficiency. $\vec{r}$ Honest responses to situations which require choice $\omega$ may, on the other hand, yield long-term advantages. $\vec{N}$ Scarcity forces societies and institutions to establish ${ }^{\bullet}$ priorities which may give rise to more efficient $\triangle$ resource use, such as devoting more resources to those $\vec{i}$ medical circumstances where the returns in terms of $\mathbb{D}$ health outcomes are likely to be the greatest. In $\frac{\overrightarrow{\mathbb{C}}}{\mathrm{O}}$ determining priorities, given the scarcity of health 3 resources, the following concepts play critical roles:

1. The principle of justice requires universal access $\$ 00$ an acceptable, decent minimum of basic health care? 2. What constitutes this acceptable, decent minimuon of basic health care will depend on the particular society's general level of affluence and other prioritieso and hence will vary not only from culture to culture buto from time to time (20). The principal task is to assess $\varrho$ other competing values and to make judgements about $\overrightarrow{\vec{O}}$ which health care needs are most pressing and which 3 responses to those needs are reasonable and $\bar{F}$ proportional.

3. When a society decides to declare a right to certain health services for all, it must incorporate into that $\Phi$ decision a willingness to give up alternative uses of $\frac{\sigma}{3}$ those resources necessary to deliver such care.

4. If medical decision-making emphasises cost- $\delta$ effective therapies, the burdens of satisfying the desired universal access will be markedly reduced. 을 5. Sometimes relatively unfettered market $\supset$ transactions can do a good job of delivering costeffective health care products and services, but, even $N_{S}$ when market processes deliver efficiency, they do so at the cost of equitable access; thus, the market-place 0 cannot be the sole determinant of access and priorities.

\section{Guidelines}

With these notions in mind, the following guidelines should be considered:

1. Society must establish the limits and the priorities for life-sustaining treatment options.

2. Processes used to establish such limits must be, an $\frac{\vec{B}}{2}$ be perceived to be, open and fair.

3. Cost effectiveness should be used whenever feasible 
to inform decisions about appropriate life-prolonging treatments in particular circumstances. Cost Effectiveness Analysis (CEA) should incorporate the best available scientific information about the results of the therapies being considered and all appropriate medical and non-medical costs and benefits -including an assessment of foreseeable changes in the patient's quality of life as a result of the proposed therapies. Although CEA is an invaluable and indispensable tool in making ethical decisions, it is not a simple formula and must not be naively applied. Several caveats are noteworthy. CEA, like all analytical frameworks, requires accurate data which may be extremely difficult and expensive to obtain. Care must be taken to be sure that the interpretation of the data is free from unwarranted extrapolations. Finally CEA must be tempered by sensitivity to human differences and caseby-case application, based on the assessment of particular benefits and burdens for individual patients. 4. CEA-based information, guidelines, and limitations should be quickly and widely distributed.

5. Society must be willing to adjust its expectations so that its selected limits become accepted practice and/or legal norms.

6. Both ethical and effective policy require that institutions conspicuously publicise any restrictive or prescriptive policy rules in advance of patient admission.

7. If institutional limits imply that physicians must deny care to some patients, they have an especially strict obligation to weigh burdens and benefits in selecting care and treatment.

8. Patients do not have a right to treatment which has no reasonable expectation of benefit.

9. If treatment is denied on the basis of a social decision, such denial must not be disguised as a medical decision.

10. When physicians believe that care is being withheld improperly, they are obliged to protest on behalf of their patients.

11. Private purchase of health care necessarily produces inequities. When the private purchase of health care significantly impinges on the fair distribution of available medical resources (as in the availability of organs for transplant) or on a society's ability to provide an acceptable, decent minimum of basic health care, such private purchase could be restricted.

\section{Dissents}

\section{Part I}

1. Requests for euthanasia by competent patients severely and irremediably suffering as a result of incurable disease may be understandable, but are not morally justified. Statutory legalisation of the intentional killing of patients by doctors is against basic morality as well as against the public interest.

Shimon Glick, MD, Beer Sheva, Israel

Arnold Rosin, MB, ChB, FRCP, Jerusalem, Israel Avraham Steinberg, MD, Jerusalem, Israel
Part II

\section{PERSISTENT VEGETATIVE STATE}

1. While it may be true that the patient with PVS has no 'self-regarding interests', it is not so obvious that no other moral interests are at stake; for example, the inherent value of life. Since patients with PVS clearly do not suffer from their state, their quality of life cannot be characterised as 'harmful' to themselves. We, therefore, cannot accept a categorical statement which rules out life-sustaining treatments.

Shimon Glick, MD, Beer Sheva, Israel

Thomas Murray, PhD, Cleveland, Ohio, USA

Arnold Rosin, MD, Jerusalem, Israel

Avraham Steinberg, MD, Jerusalem, Israel

Susan Wolf, JD, Briarcliff Manor, New York, USA

\section{Guideline working groups}

\section{Part I Competent or once competent} with advance directive

Pieter Admiraal, MD, PhD, FRSM,

The Netherlands

Gunner Dahlstrom, MD, Sweden

John Dawson, MB, BS, LMSSA, DA, MRCGP, England

Raanan Gillon, BA, MB, BS, FRCP, England

George Robertson, MD, FFARCS, Scotland

David Schiedermayer, MD, United States

Susan Wolf, JD, United States *

Stuart Youngner, MD, United States

Part II Once competent, now not competent, but without advance directive

Ronald Cranford, MD, United States

Nancy Dickey, MD, United States

Heleen Dupuis, PhD, The Netherlands

Grant Gillett, MB, ChB, DPhil, FRACS, New Zealand

Joanne Lynn, MD, MA, United States

Terrence Meece, MD, United States

Arnold Rosin, MB, ChB, FRCP, Israel

James Snyder, MD, United States *

Cees van der Meer, MD, The Netherlands *

Part III Never competent, neonates

Howard Brody, MD, United States

AGM Campbell, MB, FRCP, Scotland

Nancy Homburg, MD, United States

Dale Anne Singer, MD, United States

John Stanley, PhD, United States

Avraham Steinberg, MD, Israel

Margaret Wallace, RN, LLB, Australia *

\section{Part IV Scarcity}

Fredrick Abrams, MD, United States

Clark Boren, MD, United States

Merton Finkler, PhD, United States *

Shimon Glick, MD, Israel *

John Mielke, MD, United States

Thomas Murray, $\mathrm{PhD}$, United States

John Paris, SJ, PhD, PhL, United States

Povl Riis, MD, Denmark

Knut Erik Tranoy, PhD, MA, Norway *

^ Indicates new delegate 


\section{Notes}

(1) This international consensus statement was published earlier this year in the Fournal of the Danish Medical Association (Ugeskr laeger) 1989; 151, 11:700-706; with simultaneous summary and translation in the fournal of the Norwegian Medical Association 1989; 109:1035-1046 and the Fournal of the Swedish Medical Association 1989; $86: 1709-1712$. It is reprinted here with their permission, as a service to our international readership.

(2) Despite the wide variety of medical cultures represented, the delegates want to acknowledge that the perspectives included in the conference represented only a small fraction of the world population and did not include perspectives from Eastern Europe, the Orient, the Third World, and several other 'Western' nations, both European and American. It is our hope that the discussion provoked by the publication of these guidelines will lead to contributions from many of those perspectives.

(3) The new delegates are indicated in the list, Guideline working groups.

(4) The membership of the working groups is indicated in the same list. See Note 3.

(5) Including: The euthanasia report: report of the working party to review the British Medical Association's guidance on euthanasia. London: British Medical Association, 1988; The report of the Terminal Care Commission. Sweden: SOU, 1979:59; and The standpoint of the Royal Dutch Society for Medical Science (KNMG) concerning euthanasia. Medisch contact 1984: August.

(6) These four principles, though individually ancient, were re-articulated in the bioethics literature in the late seventies by the philosopher Thomas Beauchamp and the theologian Jaines Childress. Principles of biomedical ethics (2nd ed). Oxford: Oxford University Press, 1978, and are discussed by Gillon, R. Philosophical medical ethics. London; John Wiley and Sons, 1986. See also The Belmont report: ethical principles and guidelines for the protection of human subjects. Washington DC: DHEW pub no (os) 78-0012, 1978.

(7) Several delegates felt that 'respect for life' should be added as a fifth principle co-equal with the other four.

(8) The term competent (and all variations such as incompetent, competence, etc) is used throughout this statement in its general ethical sense indicating adequate decision-making capacity for the specific decision or class of decisions under discussion and not in its narrower legal sense.

(9) It should be remembered that in some religious traditions (for example, Orthodox Judaism) the right to refuse lifesaving treatment is not recognised as morally valid.

(10) Some delegates felt that it was not at all clear that legalising such intervention was against the public interest, especially in light of confirmation in repeated polls (most recently Roper, 1988; Harris, 1987; and A survey of opinions and experiences of Colorado physicians, 1988) that a majority of the US public and a substantial minority of physicians state that there are circumstances under which a physician should accede (or be legally permitted to accede) to a patient's request for a lethal dose of medication. Others felt that legalising such intervention was not only contrary to public interest but a violation of 'basic morality'. See dissents.

(11) Some of the delegates argued that in situations of real doubt as to whether a proposed treatment is in the patient's interest, the choice should be to withhold that treatment (the common bias toward treatment - whether resting on a technological imperative or a vitalist assumption - being unjustified). Others felt that? although physicians should never treat merely because a treatment is available, in some cases where it was not clear what the interests of the patient were, prolonging life of a quality that most patients would accept would be justified. All delegates rejected the simple vitalist? assumption that prolonging life is always in a patient's interest.

(12) Examining the rationale for the statements of family, friends, and care givers is an important part of this. process, including consideration of the possibility of conflicts of interest with the patient. Care should be taken, however, to guard against discounting the views of disagreeing parties merely because they are

(13) For cases where treatment would not be physiologically $\vec{\omega}$ futile, but nonetheless futile in the context of the whole condition, the same advice should apply as in Part $I: \mathbb{N}$ Requests for treatment.

(14) Some delegates argued that continued treatment of PVS patients can be justified by the benefit such treatment $\overrightarrow{-}$ would offer to others (for example, to parents or closed ralatives). Others argued that a PVS patient should never be treated solely for the benefit of others. Both deontological and utilitarian arguments were adduced to support this contention. Still other delegates argued that the continued treatment of a PVS patient for the benefitio of others may be justifiable if such treatment wege continued only for a short time; for example, until tei diagnosis can be established with a high degree certainty or until the family has had a reasonable amount of time to recognise the hopelessness of the situation. Sees dissents.

(15) Third person quality-of-life judgements are judgements made grammatically and logically in the third person ie, judgements about the quality of 'his' or 'her' life vis- $a$ vis first person judgements about the quality of 'my' life.

(16) This form of third person quality-of-life judgement mus be carefully distinguished from third person quality-of life judgements based on concepts of minimal sociats worth, which all delegates felt are seldom or nevero morally justifiable as a basis for medical decision-making in individual cases.

(17) It is recognised, however, that the language of benefits and burdens will not by itself resolve the most difficult dilemmas, since irreconcilable differences can always beo re-expressed in terms of a claim that their opponents have overestimated burdens and have underestimate $b$ benefits, or vice versa. These terms are nonetheless. useful in helping to focus on clinically significanfs variables and to avoid employing judgements of socia worth.

(18) A useful summary and discussion of these mechanism\& appears in Guidelines on the termination of life-sustaining treatment and the care of the dying. Hastings-on-Hudson Hastings Center, 1987:24-25.

(19) The delegates were unanimous in their feeling that this section should be regarded more as a statement in process than a final document. Some felt that for that reason it would have been better to withhold it from initial publication of these guidelines. The majority? however, felt that this version accurately represents the directions that our final deliberations will most likelyo take us and in any case will be provocative of important 
discussion of these issues and should not, therefore, be withheld from publication at this time.

(20) Cf, Securing access to health care: a report on the ethical differences in the quality of health services, Vol 1. President's Commission for the Study of Ethical Problems in Medicine and Biomedical and Behavioral Research. Washington, DC: Supt of Docs, USGPO, 1983: Ch 1:18-46.

List of co-authors of the international consensus statement John M Stanley, PhD, Edward F Mielke Professor of Ethics in Medicine, Science, and Society, Director, Program in Biomedical Ethics, Lawrence University, Appleton, Wisconsin USA

Fredrick R Abrams, MD, Associate Director, Center for Health Ethics and Policy, Assistant Clinical Professor of Obstetrics and Gynecology, University of Colorado Health Science Center, Denver, Colorado USA

Pieter V Admiraal, MD, PhD, FRSM, Senior Anaesthetist, Reinier de Graaf, Delft, The Netherlands

Clark H Boren, MD, Surgeon, Fox Valley Surgical Associates, Appleton, Wisconsin USA

Howard Brody, MD, PhD, Associate Professor of Family Practice and Philosophy, Co-ordinator, Medical Humanities Program, Michigan State University, East Lansing, Michigan, USA

AGM Campbell, MB, FRCP (Edin), Professor of Child Health, University of Aberdeen, Consultant Pediatrician, Royal Aberdeen Children's Hospital, Aberdeen, Scotland

Ronald $\mathbf{E}$ Cranford, MD, Associate Physician in Neurology, Director of Neurological Intensive Care Unit, Hennepin County Medical Center, Associate Professor of Neurology, University of Minnesota, Minneapolis, Minnesota, USA

Gunnar Dahlstrom, MD, Emeritus Professor of Medicine, University of Uppsala, Uppsala, Sweden

John Dawson, MD, MRCGP, Under Secretary, Head of Professional, Scientific, and International Affairs Division, British Medical Association, London, England

Nancy W Dickey, MD, Associate Clinical Professor of Family Medicine, University of Texas Medical School, Houston, Texas, USA

Heleen $M$ Dupuis, PhD, Professor of Bioethics, University of Leiden, Leiden, The Netherlands

Merton D Finkler, PhD, Associate Professor of Economics, Lawrence University, Appleton, Wisconsin, USA

Grant Gillett, MB, ChB, DPhil, FRACS, Neurosurgeon, Director, Clinical Ethics Program, The Medical School, University of Otago, Dunedin, New Zealand

Raanan Gillon, BA, MB, BS, FRCP, Director, Imperial College Health Service, Editor, Fournal of Medical Ethics, Visiting Professor, King's College, Centre for Medical Law and Ethics, London, England

Shimon M Glick, MD, Dean, Faculty of Health Sciences, Professor of Medicine, Ben Gurion University of The Negev, Head, Department of Internal Medicine, Soroka University Hospital, Beer Sheva, Israel

Nancy Homburg, MD, Physician in Family Practice, Medical Arts Clinic, Appleton, Wisconsin, USA

Joanne Lynn, MD, Associate Professor and Acting Director, Center for Aging Studies and Services, Co-director, ICU Research Unit, The George Washington University Medical Center, Washington, DC USA
Terrence Meece, MD, Director, Fox Valley Family Practice Residency, Associate Professor, University of Wisconsin School of Medicine, Appleton, Wisconsin, USA

John E Mielke, MD, Cardiologist, Appleton Cardiology Associates, Appleton, Wisconsin, USA

Thomas H Murray, PhD, Director, Center for Biomedical Ethics, Case Western Reserve University Medical School, Cleveland, Ohio, USA

John J Paris. SJ, PhD, PhL, Clinical Professor of Community Health, Tufts University Medical School, Adjunct Professor of Medicine, University of Massachusetts Medical School, Professor of Social Ethics, Holy Cross College, Worcester, Massachusetts, USA

Povl Riis, MD, Professor of Internal Medicine, University of Copenhagen, Editor in Chief, The Journal of the Danish Medical Association, Physician in Chief, Herlev University Hospital, Herlev, Denmark

George S Robertson, MD, FFARCS, Consultant Anaesthetist, The Aberdeen Royal Infirmary, Honorary Senior Lecturer, The University of Aberdeen, Aberdeen, Scotland

Arnold J Rosin, MB, ChB, FRCP, Chief, Department of Geriatrics, Shaare Zedek Medical Center, Professor of Medicine, Hebrew University Medical School, Jerusalem, Israel

David L Schiedermayer, MD, Internist and Clinical Ethics Instructor, Milwaukee County Hospital, Associate Director, Center for the Study of Bioethics, Medical College of Wisconsin, Milwaukee, Wisconsin, USA

Dale Anne Singer, MD, Fellow in Pediatric Hematology and Oncology, University of Michigan, Ann Arbor, Michigan, USA

James V Snyder, MD, Professor of Anesthesiology/ Critical Care Medicine, University of Pittsburgh, Associate Medical Director of Surgical Intensive Care, Medical Director of Intensive Care Unit Laboratory, Presbyterian University Hospital, Pittsburgh, Pennsylvania, USA

Avraham Steinberg, MD, Physician, Child Neurology Division, Bikkur Cholim Hospital, Physician, Department of Pediatrics, Shaare Zedek Medical Center, Head, Program of Medical Ethics, Hebrew University Hadassah Medical School, Jerusalem, Israel

Knut Erik Tranoy, PhD, Professor of Medical Ethics, Oslo University, Oslo, Norway

Cees van der Meer, MD, Emeritus Professor of Medicine, Vrije Universiteit, Amsterdam, The Netherlands

Margaret Wallace, RN, LLB, Visiting Lecturer (from Canberra College of Advanced Education, Canberra, Australia), Medical Humanities Program, Michigan State University, East Lansing, Michigan, USA

Susan $M$ Wolf, JD, Associate for Law, The Hastings Center, Briarcliff Manor, New York, USA

Stuart J Youngner, MD, Associate Professor of Psychiatry and Medicine, Faculty Member, The Center for Biomedical Ethics, Case Western Reserve University School of Medicine, Cleveland, Ohio, USA

The following delegates to the International Working Conference on Non-Treatment Decisions, May 1987, were unable to attend Guidelines for Non-Treatment Decisions: An International Working Conference, May 1988, but nonetheless, express support for the statement: Bryan Jennett, FRCP, Professor of Neurosurgery, Institute of Neurological Sciences, Southern General Hospital, Glasgow, Scotland; William J Winslade, $\mathrm{PhD}$, JD, Professor of Medical Jurisprudence, Institute for Medical Humanities at the University of Texas, Galveston, Texas. 